\title{
THPM 19.6 Multimedia ASIP SoC Codesign Based on Multicriteria Optimization
}

\author{
I-Horng Jeng, Feipei Lai, Edwin Naroska and Uwe Schwiegelshohn \\ Dept. of E.E. \& C.S.I.E., National Taiwan University and Dept. of E.T., University of Dortmund, Germany
}

\begin{abstract}
This paper presents how to codesign a multimedia ASIP (Application Specific Integrated Processor) SoC (System-onChip) to achieve a low-power and high-throughput goal for a consumer device. The codesign methodology applies Pareto's Multicriteria Optimization technique to analyze and partition the software/hardware, the instruction set and the memory hierarchy of an ASIP to provide a flexible approach of finding the best trade-off between conflicting goals based on a given weight function.
\end{abstract}

\section{INTRODUCTION}

Mobile devices will play an important if not the dominan role in future consumer electronics. The best present example for this kind of device is the cellular phone. However in the future, those devices are expected to have significantly improved multimedia capabilities. In addition they must be small, flexible and consume little power. Therefore, application-specific integrated processors (ASIP) will be a key element of those devices. Unfortunately, the goal of concurrently minimizing power consumption and maximizing throughput cannot be achieved easily as both objectives may contradict each other. For example, the battery of the commercial handheld computer usually lasts for 15 hours but less than 3 hours when connecting to the Internet. In our paper we present a codesign methodology that uses multicriteria optimization to analyze and partition the software/hardware, the instruction set and the memory hierarchy of an ASIP to provide a flexible approach of finding the best trade-off between conflicting goals based on a given weight function.

Application-specific instruction set design is an importan component of an embedded consumer device system [1] [2] [3]. It has always been addressed from the viewpoint of the ALU while the memory hierarchy was ignored. But comparing the $\left(\mathrm{P}_{\mathrm{MU}} \mathrm{D}_{\mathrm{MU}} / \mathrm{P}_{\mathrm{ALU}} \mathrm{D}_{\mathrm{ALU}}\right)$ ratios in Table 1 with $\mathrm{P}$ and $\mathrm{D}$ denoting "power" and "delay" and being modeled in [5] and [4], respectively, it shows that the memory hierarchy features [4] dominate the final cost and performance. This paper proposes the eDLX architecture to implicitly embed the memory load/store instructions implicit into the instruction addressing modes, if possible. Then the approximate improvements (upper bounds) for six client multimedia applications [3] plus the web server "HTTPD $1.52 \mathrm{a}$." are given.

\section{MULTICRITERIA OPTIMIZATION}

Pareto's MO (Multicriteria Optimization) technique maps the feasible domain in the design space into the criterion space, and computes the optimal design entry point based on the weighting objective function [7]. Two parameter groups are given for the two-level codesign MO process, see Table 1. They determine whether the process is backward compatible. The data of the first group extracted by the Sparc Architecture simulator (SAS) are analyzed for backward compatible ISA (Instruction Set Architecture), and the latter group, obtained by the GNU debugger, is for incompatible ISA. Three transformational strategies [5] are applied to the seven design candidates [3] shown in Table 2 and Fig. 1. The first is the MU optimization by RAM resource utilization such as using SRAM or embedded DRAM as main memory. The second is the ALU optimization by operation reduction and by operation substitution, e.g. SETHI (set high immediate) and emulated multiplier strategies. The final strategy is the large register memory exploited in the proposed eDLX structure. The results show that, in general, memory technology dominates the optimal trade-off. Further, ALU improvement has a significant impact on some applications, for example, Gsm requires $60 \%$ of the total execution time of multiplication. Group 2 exploits the mature on-chip SRAM technology, such as $4 \mathrm{M}$ bits CMOS SRAM with $<10$ nanosecond access time [8], to incorporate three 512K-to-19 memory addressing encoding fields into one instruction. For all applications together an estimated average speedup of 1.74 and power saving of $78.21 \%$ is achieved.

\section{THE PROPOSED EDLX SoC}

Figure 2 presents the proposed eDLX architecture that constructs a large register file for the virtual (data+stack texecution) size and a library cache memory for the $C$ library (libc.so.x). This design decodes the 512KB-register memory in every ALU instruction and eliminates unnecessary load/store instructions. It prevents pipelined load stalls while increasing the clock cycle time for large SRAM register file access. The result shows that the transferring application pair (wget and httpd) runs faster than the other encoding/decoding applications.

\section{CONCLUSION}

This paper provides an alternative method to design a future ASIP SoC suitable for multimedia applications. By incorporating the very compact embedded Linux OS, the whole multimedia system will achieve a low-power and highthroughput requirement.

\section{REFERENCES}

[1] I.-J. Huang and A.M. Despain, "Synthesis of application specific instruction sets," IEEE Trans. CAD/ICAS, vol.14, no.6, June 1995, pp. 663-675.

[2] I-Horng Jeng, Feipei Lai and Yuh-Dar Tseng, "FACE: Fine-tuned Architecture Codesign Environment for ASIP Development," Design Automation for Embedded Systems, Kluwer Academic Publishers, Volume 4, Issue 4, Oct. 1999, pp. 329-351.

[3] I-Horng Jeng and Feipei Lai, "Multicriteria Codesign Optimization for Embedded Multimedia Communication System," IEICE Trans. Fundamentals of Electronics, Communications and Computer Sciences, vol. E83A, no. 12 , Dec. 2000 , pp. 2474-2487.

[4] J.L. Henessy and D.A. Patterson, Computer Architecture A Quantitave Approach, Morgan Kaufmann Publishers, Inc., 1996.

[5] A.P. Chandrakasan, M. Potkonjak, R. Mehra, J. Rabaey, 
and R.W. Brodersan, "Optimizing power using transformations," IEEE Trans. CAD/ICA, vol.14, no.1, Jan. 1995, pp. 12-31.

[6] Steve Furber, ARM System-on-chip Architecture, Addison-Wesley, 2000.
[7] H. Eschenauer, J. Koski, A. Osyczka (ed.), Multicriteria Design Optimization: Procedures and Applications, Springer-Verlag Berlin, Heidelberg, 1990.

[8] John F. Wakerly, Digital Design Principles and Practices, Prentice-Hall International, Inc., 2000.

Table 1: Parameters for Codesign Analysis

\begin{tabular}{|c|c|c|c|c|c|c|c|c|}
\hline \multirow{2}{*}{$\begin{array}{l}\text { Multimedia } \\
\text { Application } \\
\text { Benchmarks }\end{array}$} & \multicolumn{4}{|c|}{$\begin{array}{l}\text { Parameter Group \#1 for } \\
\text { Instruction Distribution }\end{array}$} & \multicolumn{4}{|c|}{$\begin{array}{l}\text { Parameter Group \#2 for } \\
\text { Memory Distribution }\end{array}$} \\
\hline & $\frac{\mathrm{P}_{\mathrm{MU}} \mathrm{D}_{\mathrm{MU}}}{\mathrm{P}_{\mathrm{ALU}} \mathrm{D}_{\mathrm{ALU}}}$ & Mem\% & Sethi\% & Mul\% & VmData & VmStk & VmExe & VmLib \\
\hline Compress & 177.3 & 36.27 & 7.66 & 0 & 424 & 8 & 12 & 1012 \\
\hline Gzip & 549.3 & 46.03 & 4.35 & 0 & 336 & 8 & 44 & 1012 \\
\hline Wget & 306.0 & 32.30 & 4.51 & 2.92 & 60 & 16 & 80 & 1240 \\
\hline Tar & 362.8 & 35.32 & 5.50 & 0.52 & 60 & 20 & 104 & 1044 \\
\hline Gsm & 49.7 & 20.27 & 3.06 & 60.4 & 20 & 8 & 44 & 1012 \\
\hline Mpeg & 528.3 & 43.80 & 4.73 & 5.32 & 128 & 8 & 40 & 1120 \\
\hline Httpd & 511.2 & 44.66 & 1.46 & 0 & 624 & 44 & 96 & 1176 \\
\hline Average & 354.9 & 36.95 & 4.47 & 9.83 & 236 & 16 & 60 & 1088 \\
\hline Maximum & 549.3 & 46.03 & 7.66 & 60.4 & 624 & 44 & 104 & 1240 \\
\hline
\end{tabular}

p.S.: $\mathrm{Mem} \%$, Sethi \% and Mul\% indicates the percentages of the memory access, SETHI and multiply instructions.

Table 2: Transformation results for seven communication applications.

\begin{tabular}{|c|c|c|c|c|c|c|c|c|c|c|c|c|c|c|c|c|}
\hline & \multicolumn{2}{|c|}{ compress } & \multicolumn{2}{|c|}{ gzip } & \multicolumn{2}{|c|}{ wget } & \multicolumn{2}{|c|}{$\operatorname{tar}$} & \multicolumn{2}{|c|}{ gsm } & \multicolumn{2}{|c|}{ mpeg } & \multicolumn{2}{|c|}{ httpd } & \multicolumn{2}{|c|}{ Average } \\
\hline & $\mathrm{P}(\mathrm{mW})$ & $\Delta \mathrm{D} \%$ & $\mathrm{P}(\mathrm{mW})$ & $\Delta \mathrm{D} \%$ & $\mathrm{P}(\mathrm{mW})$ & $\Delta \mathrm{D} \%$ & $\mathrm{P}(\mathrm{mW})$ & $\Delta \mathrm{D} \%$ & $P(m W)$ & $\Delta \mathrm{D} \%$ & $P(m W)$ & $\Delta \mathrm{D} \%$ & $\mathrm{P}(\mathrm{mW})$ & $\Delta \mathrm{D} \%$ & $\mathrm{P}(\mathrm{mW})$ & $\Delta \mathrm{D} \%$ \\
\hline 1 & 5.965 & 0 & 8.521 & 0 & 6.189 & 0 & 6.819 & 0 & 3.853 & 0 & 8.167 & 0 & 6.752 & 0 & 6.752 & 0 \\
\hline 2 & 4.216 & 66.68 & 5.998 & 77.30 & 4.364 & 57.93 & 4.400 & 63.91 & 2.74 & 35.84 & 5.862 & 79.82 & 4.597 & 63.58 & 4.597 & 63.58 \\
\hline 3 & 5.965 & -18.14 & 8.521 & -21.84 & 6.189 & -15.97 & 6.819 & -17.62 & 3.853 & -9.8 & 8.167 & -21.52 & 6.586 & -17.48 & 6.586 & -17.48 \\
\hline 4 & 5.900 & -8.29 & 8.515 & -4.55 & 6.182 & -4.72 & 6.811 & -5.82 & 3.851 & -3.16 & 8.160 & -4.97 & 6.570 & -5.25 & 6.570 & -5.25 \\
\hline 5 & 5.965 & -8.29 & 8.521 & -4.55 & 6.189 & -4.72 & 6.819 & -5.82 & 3.853 & -3.16 & 8.167 & -4.97 & 6.586 & -5.25 & 6.586 & -5.25 \\
\hline 6 & 5.965 & 0 & 8.521 & 0 & 6.330 & -3.28 & 6.846 & -0.56 & 7.090 & -120.2 & 8.511 & -6.12 & 7.211 & -21.71 & 7.211 & -21.71 \\
\hline 7 & 1.073 & -36.27 & 1.493 & -46.03 & 1.554 & -32.3 & 1.465 & -35.32 & 2.593 & -20.27 & 1.735 & -43.8 & 0.983 & -44.66 & 1.56 & -36.95 \\
\hline & & & Group \#2 & $\begin{array}{l}\text { 1: Or } \\
3: \mathrm{En} \\
5: \mathrm{Ha} \\
\text { 7: Lar }\end{array}$ & $\begin{array}{l}\text { iginal ar } \\
\text { lbedded } \\
\text { If addres } \\
\text { ge regist }\end{array}$ & $\begin{array}{l}\text { chitectur } \\
\text { DRAM } \\
\text { s space } \\
\text { er-file st }\end{array}$ & $\begin{array}{l}\text { CSPAR } \\
\text { strategy } \\
\text { strategy } \\
\text { trategy }\end{array}$ & $\begin{array}{l}\text { (V7) } \\
\text { SETHI) }\end{array}$ & & $\begin{array}{l}\text { SRAM } \\
\text { Nord-1 } \\
\text { Multip }\end{array}$ & $\begin{array}{l}\text { rategy } \\
\text { th ext } \\
\text { strate }\end{array}$ & $\begin{array}{l}\text { ion } \\
\text { (SP }\end{array}$ & $\begin{array}{l}\text { tegy ( } \\
C \text { V } 8)\end{array}$ & THI) & & \\
\hline
\end{tabular}

Fig. 1: MO criterion space for seven multimedia applications.

Fig. 2: Pipelined eDLX architecture design.

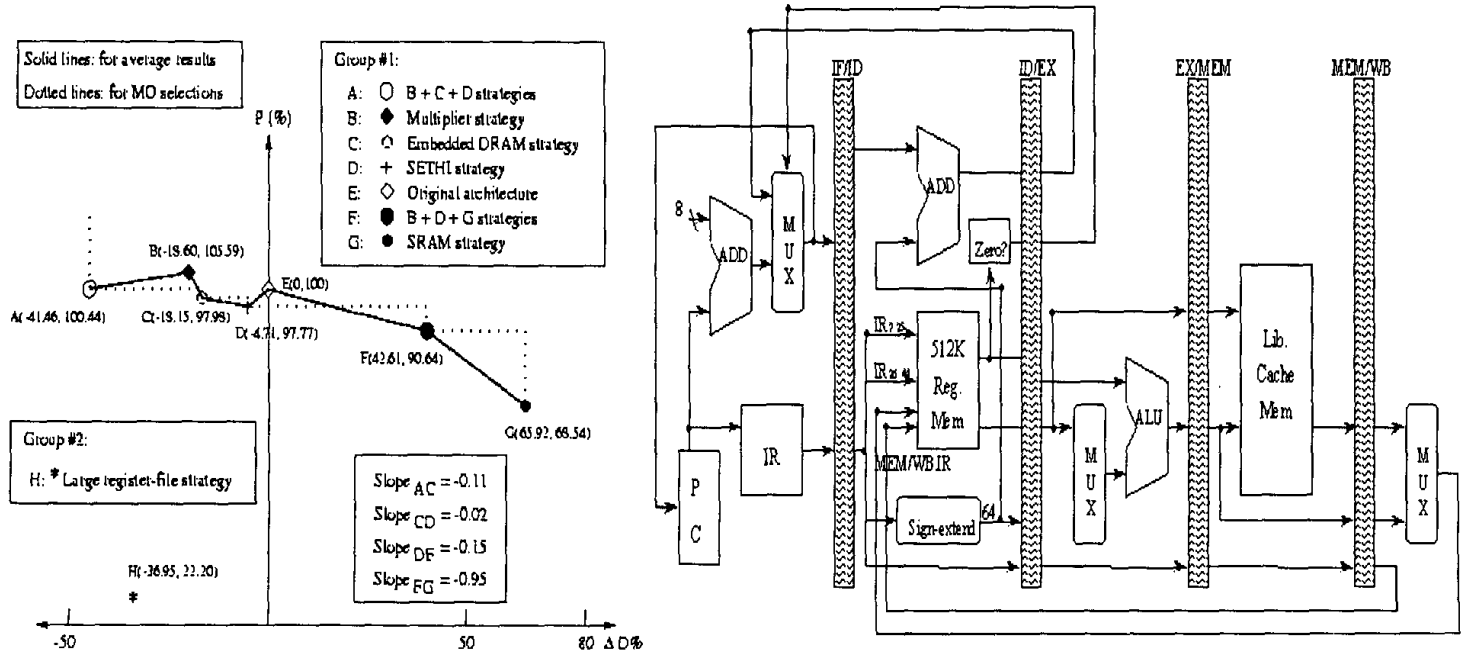

